\title{
EV Charging Recommendation Concerning Preemptive Service and Charging Urgency Policy
}

\author{
Shuohan Liu ${ }^{1}$, Yue $\mathrm{Cao}^{2}$, Wenjie Ruan ${ }^{1}$, Qiang $\mathrm{Ni}^{1}$, Michele Nati ${ }^{3}$, and Chakkaphong Suthaputchakun ${ }^{4}$ \\ ${ }^{1}$ School of Computing and Communications, Lancaster University, UK. Email: s.liu37@lancaster.ac.uk. \\ ${ }^{2}$ Research Institute of Beihang University in Shenzhen, China. Email: 871441562@qq.com. \\ ${ }^{3}$ IOTA Foundation, Germany. \\ ${ }^{4}$ Electrical and Computer Engineering Department Engineering School, Bangkok University, Thailand.
}

\begin{abstract}
Compared with traditional internal combustion engine vehicles, Electric Vehicles (EVs) have the advantage of eliminating harmful gases in the environment, with great development potential in recent years. However, because the battery capacity of EVs is limited at the current stage, where to charge (to select charging station) and when/whether to charge (order the charging priority of EVs) still limit the large-scale popularity of EVs. In this paper, we develop an Urgency First Charging (UFC) charging scheduling policy, which takes the remaining parking time and charging time of EVs as the standard of charging priority. With this, the CS benefits to the shortest trip duration (summation of travelling time through CS, and charging service time at $\mathrm{CS}$ ) is selected as optimal solution. We have conducted simulations through Helsinki's traffic scenarios. The results have shown that our proposed CS-Selection scheme effectively improves the charging comfort (in terms of waiting time and trip time) and charging efficiency (in terms of not-fully charged service due to limited parking duration).
\end{abstract}

\section{INTRODUCTION}

Electric Vehicles (EVs) have significant benefits for environmental protection, compared with traditional internal combustion engine vehicles, as they do not produce harmful gas and exhaust pollution during driving [1]. Despite these benefits, the low sustainability of EV's battery stands in the way of the broad utilization of EVs. Meanwhile, EVs require long charging time, thus optimizing the charging service to prolong the range anxiety of EVs becomes a significant problem.

Previous works [2] investigate charging scheduling (when/whether to charge), for EVs already parked at Charging Stations (CSs). When multiple EVs park at the single CS simultaneously, the CS needs to order charging priority of EVs. At the same time, the other large amount of EVs are on-the-move and need decision on where to charge (charging recommendation). Due to complex EVs motion and charging status at different CSs, navigating EVs towards a CS without a global view of all CSs' status (congestion at CS and excessive waiting time) is important.

Charging recommendation represents CS-Selection decision made for EV with charging demand. Due to the long charging time required for EV charging [3], there would be a high probability that a large number of EVs queuing at CS to wait for a charging slot, this thereby creates charging service congestion [4]. To avoid congestion, the CS-Selection scheme needs to consider both EVs' charging demand and local charging status of CSs, where an optimal solution would distribute EVs uniformly across all CSs.

The development and application of information communication technology has provided the equipment foundation for intelligent transportation systems [5], which allows information exchange among CSs and EVs [6]. A centralized manner deploys a Global Controller (GC) to aggregate CSs and EVs status for CS-Selection, which is mostly applied in the previous works [7].

In reality, EVs are not possible to stay at a fixed CS for an extremely long time, as EVs would have their independent travel plan. Previous work [7] propose deadline-driven CSSelection that considers the parking time of EVs. Here, specific parking deadline for each EV is used to simulate the specific time limitation that EVs would stay in a CS.

However, previous works are mainly based on First In First Serve (FIFS) that the EVs arrive earlier would charge earlier, and they do not consider the heterogeneity of the EVs' charging energy demand. Since EVs are supposed to get charging service before their parking deadline, those with more urgent charging demand are supposed to be scheduled with a higher priority than those not with that urgency.

In this paper, we focus on optimizing the CS-Selection scheme by jointly considering the EVs' parking duration and estimated charging scheduling when the incoming EV arrives at CSs, which would significantly reduce the possibility that EV could not get fully charged service. Instead of making FIFS as underlying charging scheduling policy, we develop an Urgency First Charging (UFC) policy, which allows incoming EV to preempt charging depending on their charging urgency (difference that energy demand minus remaining parking time). The fundamental change of this would gear an evolution design on CS-selection decision making.

\section{RELATED WORK}

Due to the substantial increase in the utilization of EVs, the CS-Selection problem has become prevalent recently. The work [8] provide a solution to select CS with a minimum estimated waiting time to avoid excessive EVs waiting at single CS. the work [9] selects CS by jointly considering the number of EVs and remaining charging time of EVs at a CS. The work [8] proves that finding the closest CS is not optimal 
selection as waiting time at CS is not considered to identify the congestion status of CS.

The other important aspect is charging scheduling when the incoming EVs arrives at CS. Nevertheless, previous works apply the FIFS policy to order charging priority of EVs. This is impractical as EV drivers may have limited time to stay at a CS. The work [10] proposes two scheduling policies: Earliest Start Time (EST) and Earliest Finish Time (EFT). In EST, the order of charging depends on the time order of EV arrival. In EFT, the order of charging depends on how early the EV could finish its charging. However, based on the limitation of parking duration that charging scheduling needs to be further considered. The work [11] proposes the CS-Selection scheme considers heterogeneous EVs. The EV with higher priority (in terms of vehicle type) is able to preempt charging service.

\section{SySTEM MODEL}

\section{A. Urgency First Charge}

UFC calculates the "charging urgency" of different EVs that parked at one CS. Charging urgency represents the negative value of the difference between charging time estimation of the EV and EV's remaining parking time.

As illustrated in Figure 1, we assume $\mathrm{EV}_{2}$ arrives later than $\mathrm{EV}_{1}$. If an charging slot becomes available at $t_{1}$, because the parking duration of each $\mathrm{EV}$ is set same, then $\mathrm{EV}_{2}$ has longer remaining parking time than $\mathrm{EV}_{1}$. Also, $\mathrm{EV}_{2}$ demands much charging energy (calculated by EV's energy difference between its full energy and its current energy) and thus larger charging time required than $\mathrm{EV}_{1}$. Here, charging urgency is calculated by remaining parking time minus charging time estimation. Then $\mathrm{EV}_{2}$ is arranged with higher charging urgency and is able to charge earlier comparing with $\mathrm{EV}_{1}$.

This policy would decrease the possibility that EV with higher charging demand and less remaining parking time could not get charging service, and would enhance the overall charging results as reducing the number of EVs miss charging.

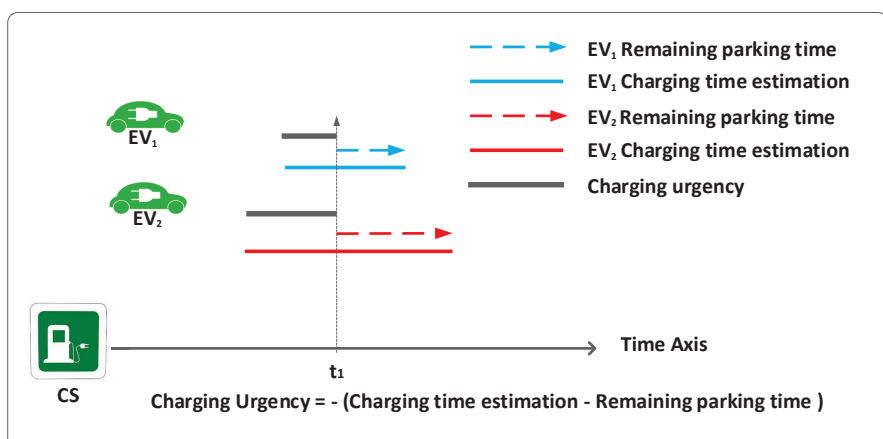

Fig. 1. Urgency First Charge

\section{B. Assumption}

In this paper, we consider a city scenario deploys a GA and several CSs. CSs are fixedly located in various parts of the city and equipped with several charging slots that allow to charge EVs in parallel. Each EV has been set a threshold SOC. The GA would globally aggregate EVs charging requests and local status of CSs, to make CS-Selection decision for EVs with charging demand.

When EV's SOC is below a threshold, EV would send its charging request to the GA, including its location, charging time. Considering each EV would only stay a limited time at $\mathrm{CS}$, each EV is addressed with a parking duration. The EVs charging scheduling is based on the UFC policy.

By jointly knowing information about CSs and parking duration, GA would be able to predict the available time to charge at each separate CS. GA would recommend CS to EV on-the-move considering the earliest available charge time at CSs, trip time from the EV's current location to CSs and trip time from CSs to the destination of EV.

\section{SySTEM DESIGN}

EVs need to consider the current charging status at CSs, and need to consider whether there are possibly preempted comparing with EVs already parked at CS $\left(N_{w}\right)$ where UFC policy would be applied. Current status at CSs would help GA to calculated estimation available charging time at the CSs. GA would make CS-Selection decision by knowing estimated available charging time together with trip time between incoming $\mathrm{EV}\left(\mathrm{EV}_{r}\right)$ to $\mathrm{CSs}$ and $\mathrm{CSs}$ to $\mathrm{EV}_{r}$ 's destination.

\section{A. Definition of Charging Urgency}

We proposed this CS-Selection scheme on the basis of underlying scheduling policy and need to know the charging scheduling of the EVs when the EV with charging demand arrives at the CS. UFC policy would be applied to reorder the charging priority of EVs. If an incoming EV has a higher value of charging urgency than parked EVs, the UFC policy allows this EV to preempt charging, before those EVs been parked. The charging urgency is given by:

$$
V_{e v}=-\left(T_{e v}^{a r r}+D_{e v}-T_{e v}^{s t a}-T_{e v}^{c h a}\right)
$$

Here, $T_{e v}^{a r r}$ reflects the EV's arrival time, $D_{e v}$ reflects the parking duration EVs parked at one CS, $T_{e v}^{s t a}$ reflects the time EV has spent at the CS after its arrival. $T_{e v}^{c h a}$ reflects EV's charging time, which would be detailed in the following section.

\section{B. Estimation of Available Charging Time at CS}

The time to finish charging of those EVs currently charging simultaneously on each charging slot, is defined as estimated available time to charge $\left(\mathrm{EATC}_{c s}\right)$. Each CS has $\delta$ charging slots giving parallel charging services.

In Algorithm 1, we detail the implementation of the estimation available time to charge at each CS. Current time $T_{\text {cur }}$ represents the time in the network. The operations between lines 1 and 3 denote no EV is under charging at the CS, the arrival time of the $\mathrm{EV}_{r}$ (the on-the-move EV needs to charge), $T_{e v_{(r)}}^{a r r}$, would be returned as the available time to charge at this $\mathrm{CS}$. Here, $T_{e v_{(r)}}^{a r r}$ reflects the time that the $\mathrm{EV}_{r}$ reaches at the $\mathrm{CS}$, which is calculated by adding travel time towards the CS 


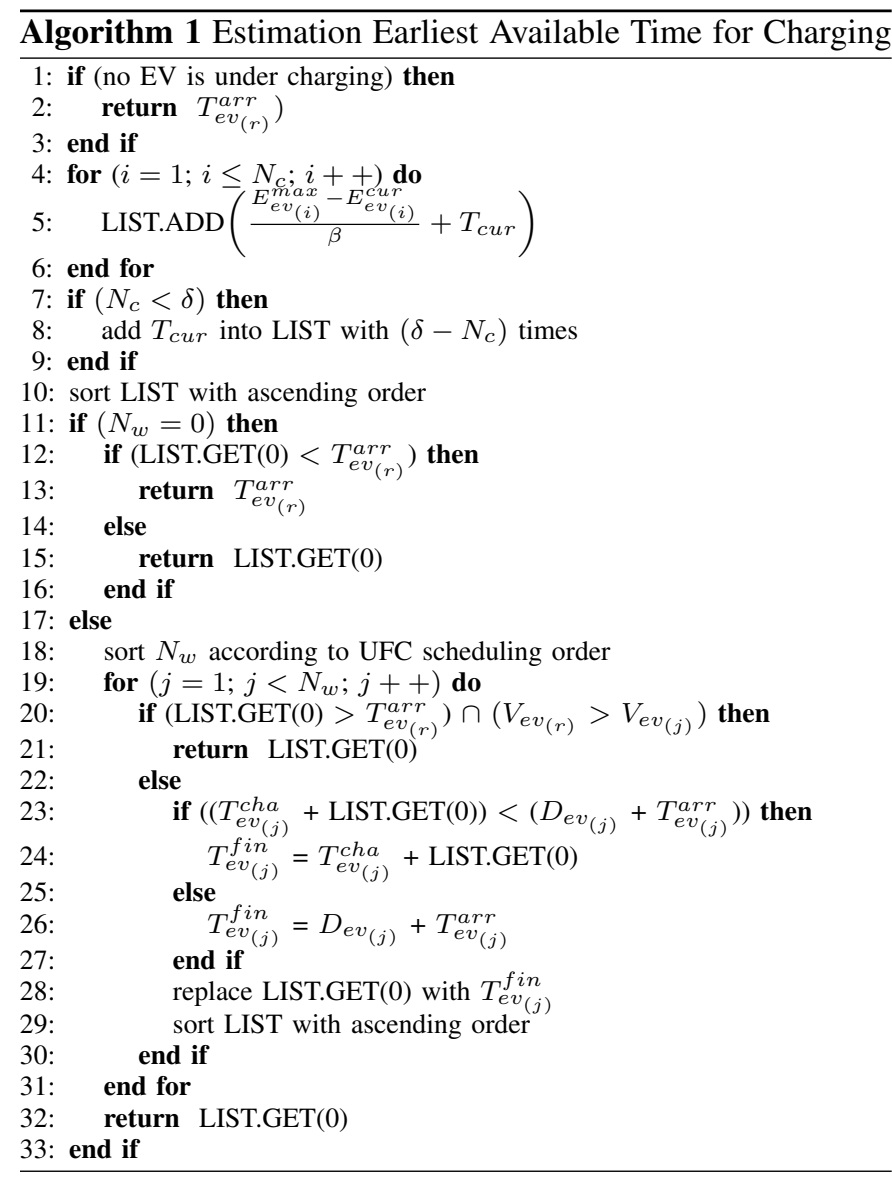

from the $\mathrm{EV}_{r}$ 's current location to the current time slot of the simulation environment:

$$
T_{e v(r)}^{a r r}=T_{c u r}+T_{e v}^{t r a}(r)
$$

Furthermore, LIST is set to reflect available time to charge at each slot (EVs finish current charging). Line 5 implies $\frac{E_{e v}^{\max }-E_{e v}^{c u r}(i)}{\beta}+T_{c u r}$ to reflect the finish time that $\mathrm{EV}_{i}$ in $N_{c}$ full recharged its battery. The fully recharging time and $T_{c u r}$ would be summed as the available time to charge at charging slot that $\mathrm{EV}_{i}$ is currently charging. The following lines 7 to 9 add $T_{\text {cur }}$ into LIST with $\left(\delta-N_{C}\right)$ times, which denotes that number of $\left(\delta-N_{C}\right)$ slots at this CS are available to provide charging service from $T_{\text {cur }}$ as they are not occupied. Line 10 sorts LIST as ascending order so that the first available charging slot would be at the top of LIST.

It needs to consider the scheduling situation when $\mathrm{EV}_{r}$ arrives at $\mathrm{CS}$ in the process of CS-Selection. If there is no $\mathrm{EV}$ parked at CS waiting for charging (the condition between lines 11 and 16). $\mathrm{EV}_{r}$ would get charging service without charging urgency comparison with $N_{w}$ when it arrives at the $\mathrm{CS}$. Line 12 considers the condition $\mathrm{EV}_{r}$ arrives later than LIST.GET(0), where LIST.GET(0) indicates the first available charging slot at the CS, it would return $T_{e v_{(r)}}^{a r r}$ as available time to charge at this CS because CS would have a charging slot available upon arrival. Or $\mathrm{EV}_{r}$ needs to wait until the first charging slot available, which would return LIST.GET(0) as the available charging time at this CS (lines 14 to 15).

Line 17 considers the other condition different from line 11 that there are EVs waiting at the CS. Line 18 sorts $N_{w}$ firstly according to the UFC scheduling policy. A loop operation for $\mathrm{EV}_{j}$ (waiting EVs in the queue of $N_{w}$ ) is given between lines 19 and 31. The UFC policy would take into account the possibility of preemption. Line 20 is designed to determine the charging order between the parked EVs and $\mathrm{EV}_{r}$ :

- Line 20 denotes $\mathrm{EV}_{r}$ arrives earlier than LIST.GET(0) and has a higher charging urgency than $\mathrm{EV}_{j}$, then $\mathrm{EV}_{r}$ is allowed to preempt charging before the parked EVs. As $\mathrm{EV}_{r}$ and $\mathrm{EV}_{j}$ has different identities of charging energy demands. Their $T_{e v}^{c h a}$ would be calculated separately. As $\mathrm{EV}_{j}$ has been parked, its $T_{e v}^{c h a}$ is given by:

$$
T_{e v(j)}^{c h a}=\frac{E_{e v}^{\max }-E_{e v}^{c u r}}{\beta}
$$

As $E_{r}$ is currently travelling, extra energy would be consumed that $\mathrm{EV}_{r}$ moving from its current location to the selected CS, which would be calculated as $\left(S_{e v} \times\right.$ $\left.T_{e v_{(r)}}^{t r a} \times \alpha\right)$. So its $T_{e v}^{c h a}$ is given by:

$$
T_{e v(r)}^{c h a}=\frac{E_{e v_{(r)}}^{\max }-E_{e v(r)}^{c u r}+\left(S_{e v} \times T_{e v}^{t r a} \times \alpha\right)}{\beta}
$$

- Apart from the condition at line 20, Algorithm 1 considers the other condition. From lines 22 to 30, it considers that $\mathrm{EV}_{r}$ has not arrived at the CS when the first charging slot available (LIST.GET(0) $<T_{e v_{(r)}}^{a r r}$ ), or the charging urgency of $\mathrm{EV}_{r}$ is lower than the charging urgency of $\mathrm{EV}_{j} . \mathrm{EV}_{j}$ would charge its battery when the first charging slot is available. Lines 24 and 26 calculate the charging finish time $T_{e v_{(j)}}^{f i n}$ of $\mathrm{EV}_{j}$ under different condition.

If $\mathrm{EV}_{j}$ has enough remaining parking time (condition under line 23), $T_{e v_{(j)}}^{f i n}$ would be calculated by summing the first available charging time LIST.GET(0) with $T_{e v_{(j)}}^{c h a}$ as $\mathrm{EV}_{j}$ would be fully charged. Line 26 considers that $\mathrm{EV}_{j}$ could not be fully charged before it departure.

LIST.GET(0) would then be replaced by $T_{e v_{(j)}}^{f i n}$ as current available charging time at this charging slot (line 28). LIST would be sorted later according to ascending order, so that LIST.GET(0) would be updated and be the first available charging slot for further calculation.

After the above loop operation finishes and every $\mathrm{EV}_{j}$ is sorted. Line 32 returns LIST.GET(0) as the earliest available time to charge.

\section{CS-Selection Decision Making}

With Algorithm 1, we are able to obtain estimated available time to charge at each CS $\left(\mathrm{EATC}_{c s}\right)$. In order to shorten overall trip time of $\mathrm{EV}$ drivers, the following aspects need to be considered as influence factors when selecting CS:

- The traveling time from the $\mathrm{EV}_{r}$ 's current location to the selected CS, which is denoted as $T_{e v_{(r)}}^{t r a}$.

- The estimation duration time staying at the selected CS would be constrained by $D_{e v_{(r)}}$. 
- The estimated travelling time from the selected CS to $\mathrm{EV}_{r}$ 's destination $T_{c s, d}^{\text {min }}$.

$T_{e v_{(r)}}^{c s, d}$ is defined by summing the above inputs and is given by:

$$
T_{e v}^{c s, d}=T_{e v_{(r)}}^{t r a}+\mathrm{EATC}_{\mathrm{cs}}+T_{e v_{(r)}}^{c h a}+T_{c s, d}^{m i n}
$$

However, if $\left(T_{e v_{(r)}}^{c h a}+\mathrm{EATC}_{\mathrm{cs}}\right)$ is larger than $D_{e v_{(r)}}$, then $\mathrm{EV}_{r}$ is not able to fully recharge before its parking duration deadline, and $T_{e v(r)}^{c s, d}$ would be given by:

$$
T_{e v(r)}^{c s, d}=T_{e v_{(r)}}^{t r a}+D_{e v_{(r)}}+T_{c s, d}^{m i n}
$$

By generating $T_{e v(r)}^{c s, d}$ of each CS, the GA would select the CS with minimum $T_{e v(r)}^{c s, d}$ to $\mathrm{EV}_{r}$ as to shorten the overall trip time of EV and improve degree of charging QoE.

\section{Performance Evaluation}

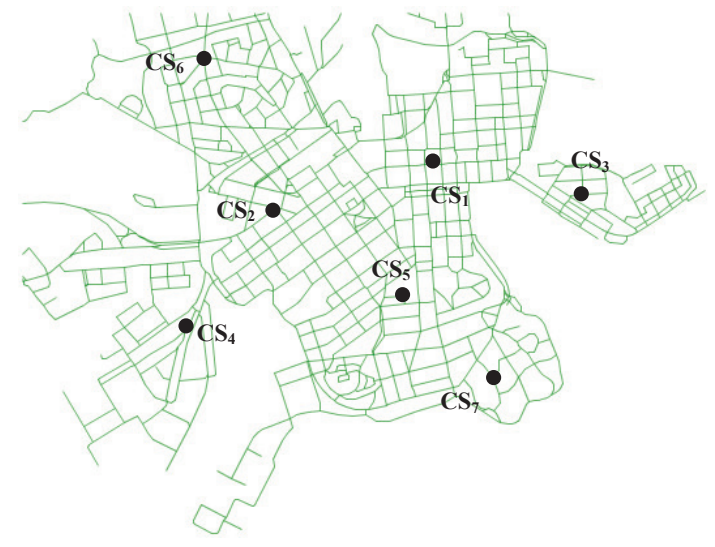

Fig. 2. Simulation Scenario of Helsinki City

\section{A. Simulation Setting}

We set up a scenario in Opportunistic Network Environment (ONE) [12] to simulation the entire EV charging system. In Figure 2, We used Helsinki, Finland for urban environment simulation, the entire city scenario is with an area of $4500 \times 3400 \mathrm{~m}^{2}$. During the simulation, we set a random destination for each EV to avoid EVs from clustering at a specific location. The paths of the EVs to their destinations are calculated via Dijkstra algorithm. At the same time, each EV is also set with SOC, which are used as the flag to start sending the charging request. EVs with $[30 \sim 50]$ $\mathrm{km} / \mathrm{h}$ variable moving speed are initialized in the network. The configuration of EVs follows the charging specification: Electricity Capacity, Max Travelling Distance, SOC threshold. We configure the EVs using Coda Automotive [13] \{33.8 $\mathrm{kWh}, 193 \mathrm{~km}\}$ average energy consumption $0.1751 \mathrm{kWh} / \mathrm{km}$ with three different SOC: $30 \%, 35 \%$ and $50 \%$.

Three CS-Selection scheme would be compared: Proposed: The GA applies UFC policy in scheduling prediction of CSs and selects the CS with minimum trip duration which is detailed in section IV; Minimum Waiting Time (MWT): The GA applies FIFS policy in scheduling. CS would let parked
EVs charging earlier when the incoming EV arrives. The GA selects the CS with minimum waiting time in scheduling prediction; Minimum Distance (MD): the GA selects the CS with minimum distance to the incoming EV, that is selecting the CS that is closest to the incoming EV.

A 12 hours' duration scenario is simulated with a $0.1 \mathrm{~s}$ resolution. EVs' positions and energies are updated in each resolution, no matter EVs are under charging or travelling. We deployed 6 CSs in the city, each of them is with large enough electric energy and is equipped with 5 charging slots that provide $62 \mathrm{~kW}$ charging power.

We set three evaluation metrics: Number of Times EVs Could Not Fully Charged is evaluated as the performance metric, which counts the times of EVs arrive at CSs but leave before it get fully charged due to parking duration limitation. Average Charging Waiting Time measures the average time spends between the time EVs arrives at CSs and the time EVs charges. Average Trip Duration reflects the entire time period spends from the incoming EV's original location to its destination, which contains its charging time and trip time.

\section{B. Performance Analysis}

We deploy $150 \mathrm{EVs}$ to observe the influence of parking duration changes in Figure 3. The proposed scheme achieves the lower times that EVs could not fully charged than MWT and MD in Figure 3(a), as the underlying UFC policy in proposed scheme considers charging urgency of different EVs and allows EV with higher charging urgency preempt charging. Meanwhile, the proposed scheme achieves the shortest average waiting time in all three CS-Selection schemes as illustrated in Figure 3(b), because the proposed scheme predicts the scheduling that the incoming EV arrives at each CS, which distributes charging service of each CS more balanced, and it is beneficial to reduce average waiting time of EVs. Figure 3(c) demonstrates the performance of three CS-Selection schemes on trip duration, it could be found that with the parking duration increases, the proposed scheme has a shorter average trip duration than MWT and MD. This is because as the parking duration increases, more charging preempt might occur.

We set a same parking duration 1500s to observe the performance of three different CS-Selection schemes when the total number of EVs changes in Figure 4. When the proportion of EVs and CSs is small, the proposed scheme has less number of times that EVs could not fully charged over MWT and MD. As the proportion of EVs-CSs increases, CSs are more likely to have a large amount of charging congestion. At this time, the proposed scheme performs much better as illustrated in Figure 4(a). In Figure 4(b), the proposed scheme achieves a shortest average waiting time. It is worth mentioning that when there are $240 \mathrm{EVs}$ in the network, the average waiting time of all three schemes decreases. This is because the congestion is more severe than it in $150 \mathrm{EVs}$ and $180 \mathrm{EVs}$ scenario, the overall times of charging service CSs provided is decreased. So the average waiting time to complete charging also decreased. In $240 \mathrm{EVs}$ scenario, it shows the proposed scheme has a 


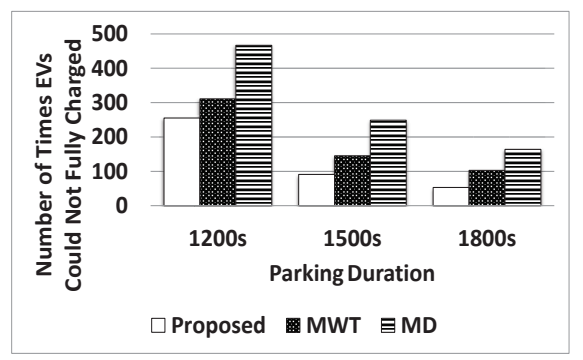

(a) Number of Times EVs Could Not Fully Charged

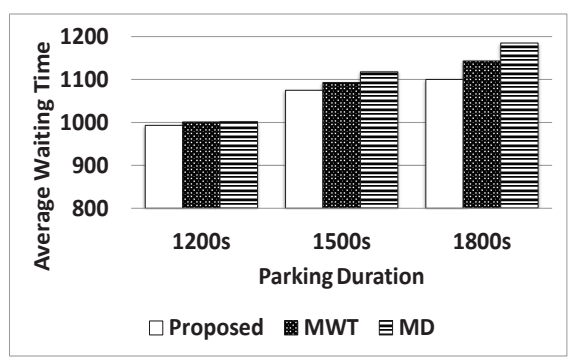

(b) Average Charging Waiting Time

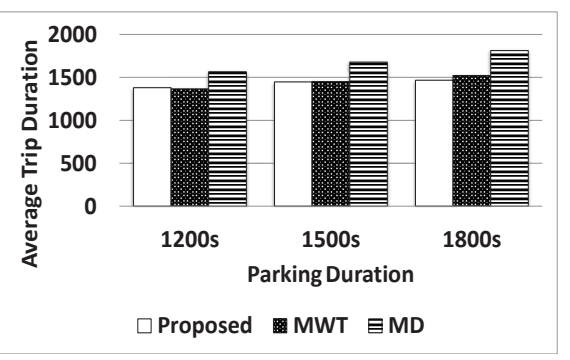

(c) Average Trip Duration

Fig. 3. Simulation Result Changing Parking Duration

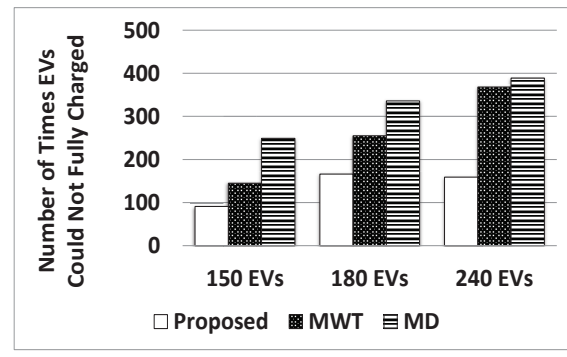

(a) Number of Times EVs Could Not Fully Charged

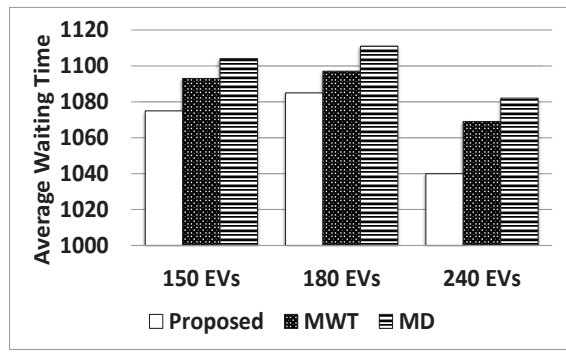

(b) Average Charging Waiting Time

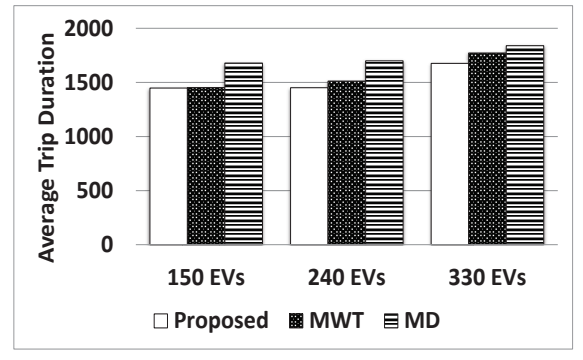

(c) Average Trip Duration

Fig. 4. Simulation Result Changing Number of EVs

shorter average charging time when congestion occurs. The proposed scheme also has the shortest trip duration among the all three CS-Selection scheme in Figure 4(c). As the total number of EVs increases, more preempt charging might occur, and the proposed performance even better than MWT and MD.

\section{CONCLUSION}

In this paper, we propose a CS-Selection scheme considering charging urgency of EVs. The selection computation predicts the charging scheduling when the incoming EV arrives at the CS using UFC policy, which is different from FIFS policy that considers EVs' individual energy demand and parking duration. The EV with higher energy demand and shorter remaining parking time is allowed to preempt charging comparing with parked EVs. The evaluation under the Helsinki city scenario confirmed the performance of our proposed CS-Selection scheme, which could significantly decrease the possibility the incoming EVs could not get fully charged. Our proposed CS-Selection scheme helps to improve the QoE of EV drivers, it also provides an EVs charging urgency-driven idea for further CS-Selection scheme.

\section{ACKNOWLEDGE}

The research is supported in part by the Joint Fund of Guangdong Province Foundation and Applied Science (2019A1515110238).

\section{REFERENCES}

[1] J. Brady and M. O'Mahony, "Travel to work in dublin. the potential impacts of electric vehicles on climate change and urban air quality," Transportation Research Part D: Transport and Environment, vol. 16, pp. 188-193, 032011.
[2] J. C. Mukherjee and A. Gupta, "A review of charge scheduling of electric vehicles in smart grid," IEEE Systems Journal, vol. 9, no. 4, pp. 15411553, Dec 2015.

[3] V. T. Tran, D. Sutanto, and K. M. Muttaqi, "The state of the art of battery charging infrastructure for electrical vehicles: Topologies, power control strategies, and future trend," in 2017 Australasian Universities Power Engineering Conference (AUPEC), Nov 2017, pp. 1-6.

[4] E. S. Rigas, S. D. Ramchurn, N. Bassiliades, and G. Koutitas, "Congestion management for urban ev charging systems," in 2013 IEEE International Conference on Smart Grid Communications (SmartGridComm), Oct 2013, pp. 121-126.

[5] G. Dimitrakopoulos and P. Demestichas, "Intelligent transportation systems," IEEE Vehicular Technology Magazine, vol. 5, no. 1, pp. 77-84, March 2010.

[6] Y. Cao, H. Song, O. Kaiwartya, A. Lei, Y. Wang, and G. Putrus, "Electric vehicle charging recommendation and enabling ict technologies: Recent advances and future directions," 2017.

[7] Y. Cao, T. Wang, O. Kaiwartya, G. Min, N. Ahmad, and A. H. Abdullah, "An ev charging management system concerning drivers' trip duration and mobility uncertainty," IEEE Transactions on Systems, Man, and Cybernetics: Systems, vol. 48, no. 4, pp. 596-607, April 2018.

[8] M. Gharbaoui, L. Valcarenghi, R. Bruno, B. Martini, M. Conti, P. Castoldi, S. Scuola, C. anna, C. Iit, G. Via, and Moruzzi, "An advanced smart management system for electric vehicle recharge," 032012.

[9] H. Qin and W. Zhang, "Charging scheduling with minimal waiting in a network of electric vehicles and charging stations," 09 2011, pp. 51-60.

[10] M. Zhu, X. Liu, L. Kong, R. Shen, W. Shu, and M. Wu, "The charging-scheduling problem for electric vehicle networks," in 2014 IEEE Wireless Communications and Networking Conference (WCNC), April 2014, pp. 3178-3183.

[11] Y. Cao, S. Liu, Z. He, X. Dai, X. Xie, R. Wang, and S. Yu, "Electric vehicle charging reservation under preemptive service," in 2019 1st International Conference on Industrial Artificial Intelligence (IAI), July 2019, pp. 1-6.

[12] A. Keränen, J. Ott, and T. Kärkkäinen, "The ONE Simulator for DTN Protocol Evaluation," in ICST SIMUTools' 09, Rome, Italy, March, 2009.

[13] [Online]. Available: www.codaautomotive.com. 